\title{
De l'utilisation du phénomène d'injection dans un évacuateur de crues
}

\author{
Application au barrage de l'Iril Emda
}

\section{On the use of the ejection phenomenon in a flood spillway}

\author{
Application to the Iril Emda Dam
}

\author{
PAR J. CHABERT \\ INGÉnIEUR aU Laboratoire National D'HydrauliQue \\ English synopsis, p. 115.
}

\section{I. - INTRODUCTION}

Le phénomène d'injection joue un rôle important dans les conditions de fonctionnement du dispositif de dispersion de l'évacuateur de crues du barrage de l'Iril Emda qui a été étudié sur modèle au Laboratoire National d'Hydraulique. Nous avons voulu signaler ici la bonne concordance que nous avons constatée entre la prévi- sion théorique de ses effets à partir des théories de Tollmien et les mesures expérimentales effectuées sur le modèle, malgré les hypothèses simplificatrices que nous avons dû admettre pour assimiler ce cas relativement complexe au schéma très simplifié de la théorie.

\section{II. - CARACTÉRISTIQUE DU BARRAGE ET DE L'ÉVACUATEUR DES CRUES}

Le barrage de l'Iril Emda est situé dans le département de Constantine à $80 \mathrm{~km}$ de Bougie. Il est installé immédiatement à l'aval du confluent des deux oueds Embarek et Berd dont la jonction constitue l'oued Agrioun et est destiné à l'accumulation d'une importante quantité d'eau (155 millions de $\mathrm{m}^{3}$ ) pour l'alimentation de deux usines hydro-électriques placées en série.

Ce barrage est du type barrage poids en enrochements, solution qui a été retenue pour tenir compte du comportement médiocre du terrain (fig. 1). Il comprend, en son centre, un massif en béton supportant à sa partie su- périeure un déversoir muni de trois pertuis. Chacun des pertuis, qui peut être obturé par une vanne secteur, débite dans une galerie de section ovoïde inscriptible dans un carré de $10 \mathrm{~m}$ de côté, l'écoulement s'effectuant à air libre à l'intérieur de ces galeries.

Le fond du lit est au voisinage de la cote 460 et la crête du barrage à la cote 535 . Sous la retenue normale (cote 532) l'évacuateur de crue peut évacuer un débit́t de $2.500 \mathrm{~m}^{3} / \mathrm{s}$. La cote du seuil déversant est à 520 , celle du radier à l'aval de l'évacuateur de crues est à 460,50 . La dénivellation est done de $59,50 \mathrm{~m}$.

Le profil en long de l'évacuateur de crues 


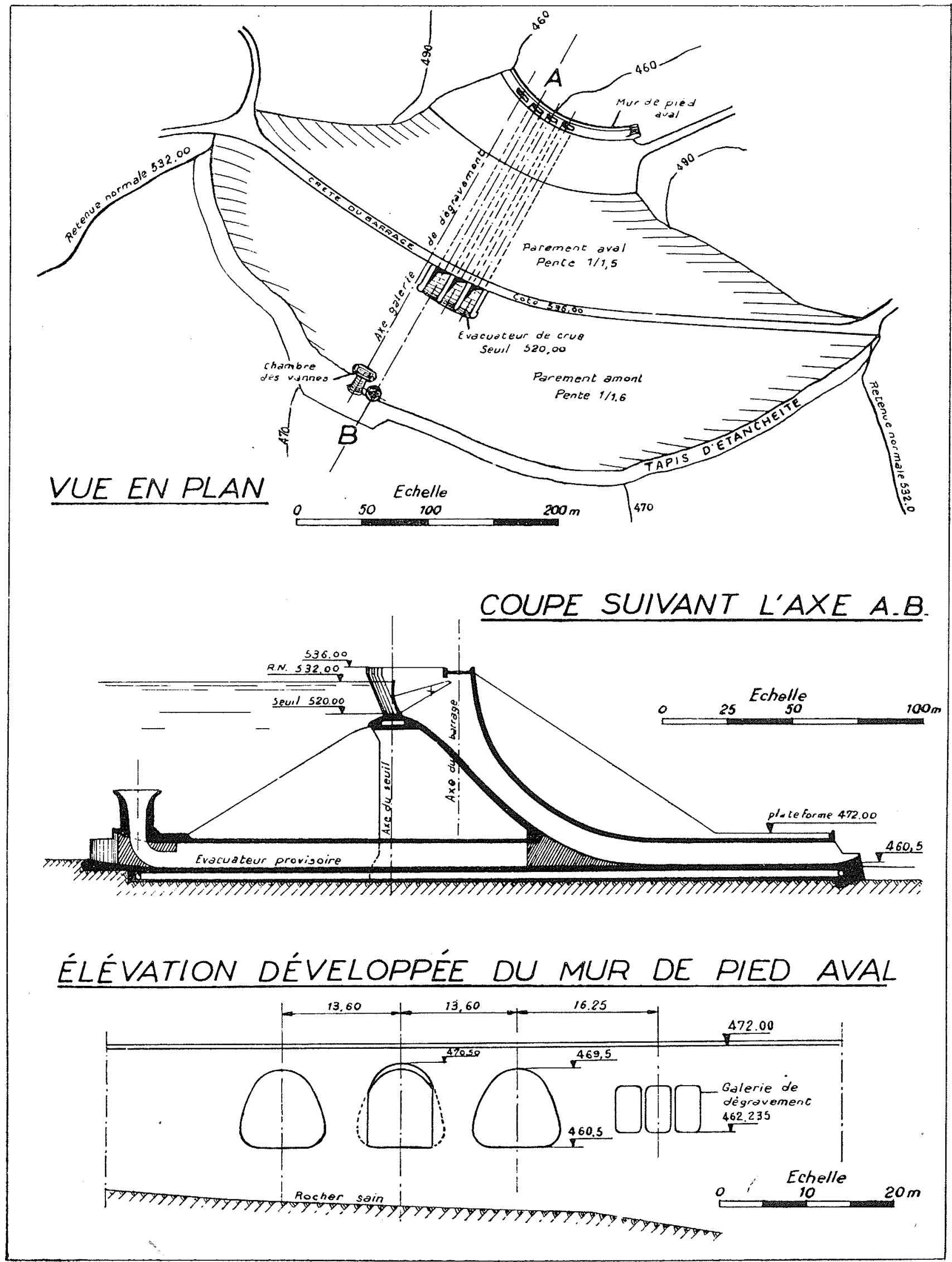

FIG. 1. 

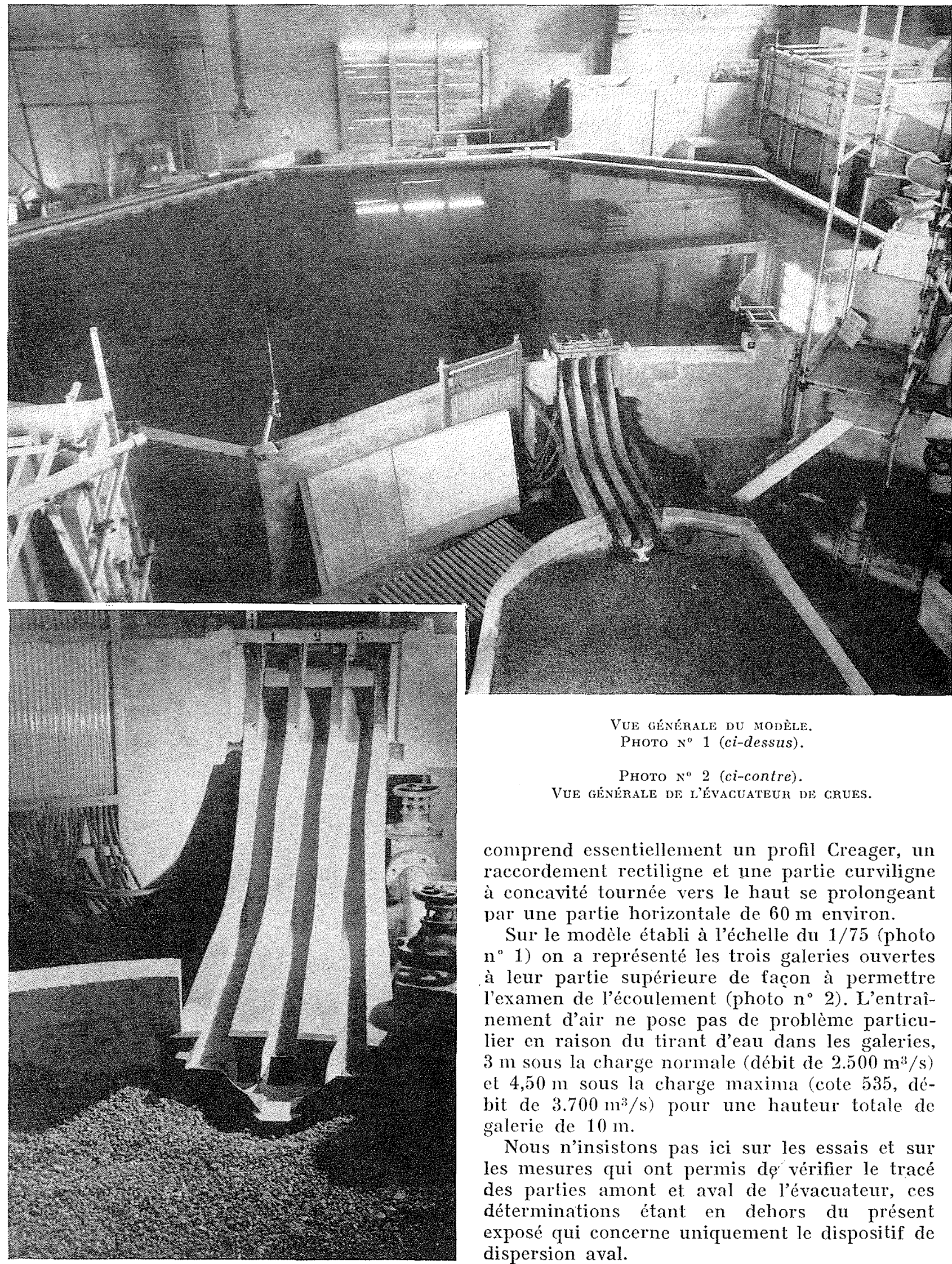

VUE GÉNERALE DU MODÈLE.

Рното $\mathrm{N}^{0} 1$ (ci-dessus).

Photo $\mathrm{N}^{\mathrm{a}} 2$ (ci-contre)

VUE GÉNÉRALE DF L'ÉVACUATEUR DE CRUES.

comprend essentiellement un profil Creager, un raccordement rectiligne et une partie curviligne à concavité tournée vers le haut se prolongeant par une partie horizontale de $60 \mathrm{~m}$ environ.

Sur le modèle établi à l'échelle du $1 / 75$ (photo $\mathrm{n}^{\circ} 1$ ) on a représenté les trois galeries ouvertes à leur partie supérieure de facon à permettre l'examen de l'écoulement (photo $n^{\circ} 2$ ). L'entraînement d'air ne pose pas de problème particulier en raison du tirant d'eau dans les galeries, $3 \mathrm{~m}$ sous la charge normale (débit de $2.500 \mathrm{~m}^{3} / \mathrm{s}$ ) et $4,50 \mathrm{~m}$ sous la charge maxima (cote 535 , débit de $3.700 \mathrm{~m}^{3} / \mathrm{s}$ ) pour une hauteur totale de galerie de $10 \mathrm{~m}$.

Nous n'insistons pas ici sur les essais et sur les mesures qui ont permis dé vérifier le tracé des parties amont et aval de l'évacuatemr, ces déterminations étant en dehors du présent exposé qui concerne uniquement le dispositif de dispersion aval. 


\section{III. - DISPOSITIF DE DISPERSION}

\section{a) Considérations générales.}

L'absence de toute dénivellation entre le radier de la sortie des galeries et le lit aval conduit dans le cas du fonctionnement d'un seul pertuis à un écoulement à peine dénoyé à l'aval. Le plan d'eau s'établit en effet à $2 \mathrm{~m}$ au-dessus de ce radier, remplissant ainsi les galeries qui ne sont pas en service du fait des tirants d'eau nécessaires pour évacuer $830 \mathrm{~m}^{3} / \mathrm{s}$ dans la vallée, et de la surélévation du lit résultant de la formation d'une barre à l'aval de la zone d'impact du jet.

Dans ces conditions, lorsqu'un pertuis est déjà en fonctionnement, la mise en service d'un autre pertuis conduit à la formation d'un important ressaut dans la partie aval de la galerie correspondante par suite de la masse d'eau inerte chassée violemment vers l'aval.

Ce ressaut entraine une diminution considérable du tirant d'air dans la galerie et risque de la mettre momentanément en charge, ce qui peut être préjudiciable à la bonne tenue des ouvrages. Il est done nécessaire d'étudier un dispositif de dispersion qui réalise les conditions suivantes :

Tout d'abord permettre une diffusion suffisante des jets dans l'air par augmentation de la portée naturelle de ceux-ci, et ensuite un bon étalement des zones d'impact pour réduire les affouillements et les reporter loin du mur de pied des ouvrages; enfin, étant donné les conditions particulières de l'implantation de l'ouvrage, le. dispositif devra permettre et ne pas gêner la vidange des galeries qui ne sont pas en service.

\section{ETUDE DES DIFFERENTS DISPOSITIFS DE DISPERSION ESSAYÉS}

De nombreux dispositifs de dispersion ont été étudiés; on ne reprendra pas ici cette étude en

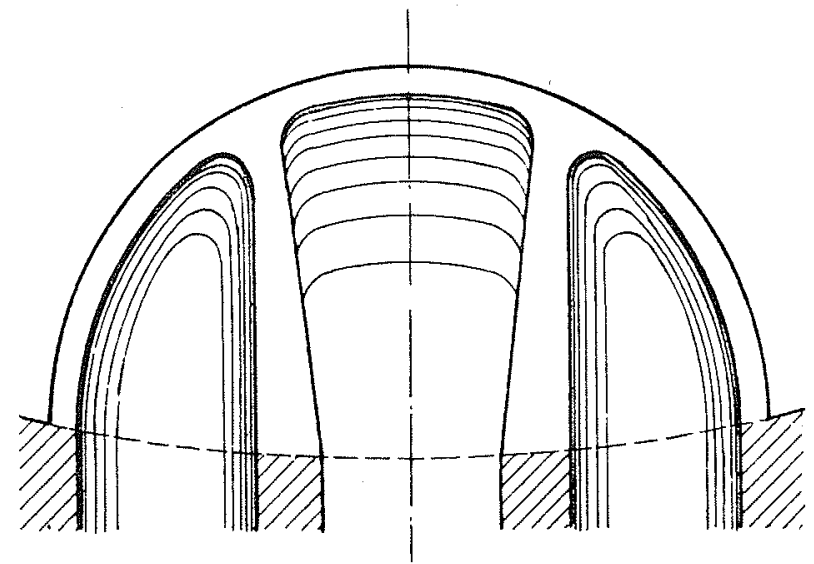

FIG. 2. détail, mais on indiquera seulement les quelques étapes qui ont marqué l'évolution des for-

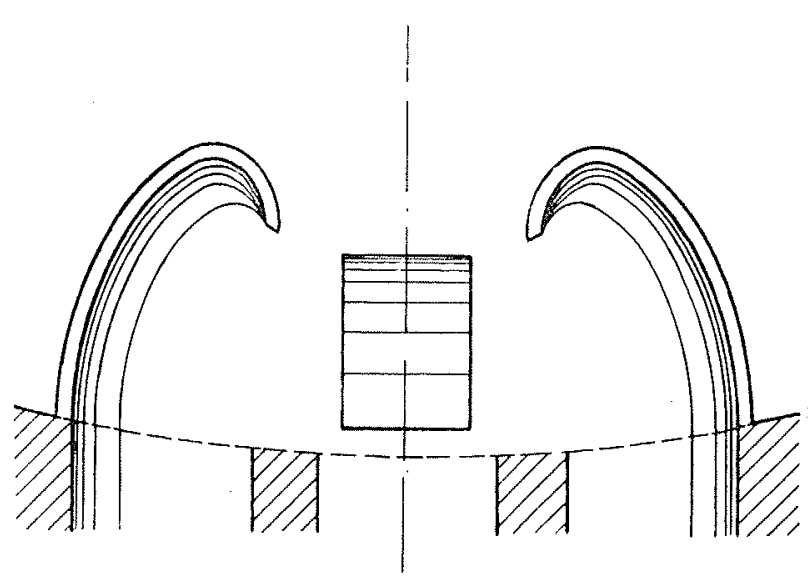

FIG. 3.

mes jusqu'au dispositif de dispersion qui a étẻ finalement retenu.

Après quelques essais préliminaires de dents de dispersion difficilement adaptables si on les utilise seules à cet évacuateur de crues, un premier dispositif, formé de trois augets, a été étudié (fig. 2) : la solution est valable aux gros débits, mais on observe des tourbillons importants dans les augets des galeries non en service où un important tirant d'eau demeure; la mise en fonctionnement des pertuis correspondant en est de ce fait rendue très difficile.

L'auget central a été alors remplacé par une dent de dispersion (fig. 3) : le fonctionnement est nettement amélioré, mais il est nécessaire de travailler de très près les formes des augets

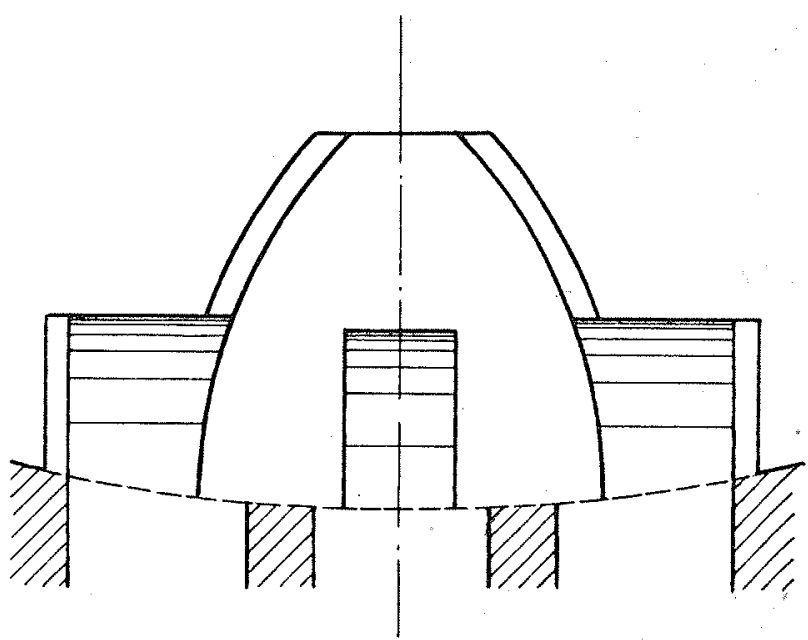

FIG, 4. 
pour éviter les réllexions nuisibles des filets liquides provenant du jet central.

Les augets latéraux ont été ensuite remplacés, pour des raisons hydrauliques et économiques, par des dents de dispersion prolongées par une petite murette conduisant à un ouvrage de forme plus simple (fig. 4). L'éturle des formes à donner à ces murettes a conduit à réaliser des surfaces conoïdales et par conséquent réglées, permettant de diffuser suffisamment les jets en évitant des réflexions gènantes.

Ce sont les études de détails effectuées à partir de ce dernier type de dispositif qui ont permis de préciser les formes exactes à donner aux ouvrages et de déterminer ainsi le dispositif de dispersion définitif.

\section{b) Description du dispositif de dispersion.}

Le principe de ce dispositif de dispersion est le suivant: le débit de chaque galerie est partagé en deux, la fraction la plus importante étant diffusée par un tremplin profilé pour obtenir une portée suffisante d'un jet devant de plus être aéré.

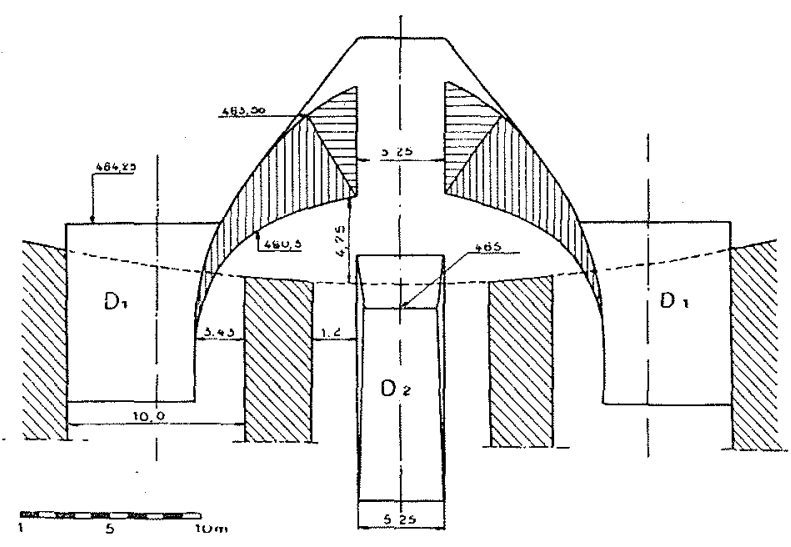

Fig. 5 .

Le reste du débit est utilisé pour vider par injection s'ils ne fonctionnent pas, les deux autres pertuis ef éviter les rentrées d'eau par l'aval quel que soit celui des trois pertuis qui fonctionne. Mais cette fraction du débit ne doit pas créer d'affouillement à l'aval du dispositif de dispersion, elle dọit par conséquent être reprise par un ouvrage arasé à une cote ne gênant pas l'effet d'injection.

Le dispositif est représenté par la figure 5 et les photos $n^{\circ} 3$ et 4 . Les deux dents $D_{1}$ et la dent $D_{2}$ sont des surfaces cylindriques dont les angles du plan tangent à l'arête extrême avec le plan horizontal sont respectivement egaux à $25^{\circ} 30^{\prime}$ et $40^{\circ}$. Les jets non diffusés par les dents sont repris par deux bees qui pour simplifier les coffrages sont des surfaces réglées. Leur pente est déterminée pour éviter que les jets ne les heurtent trop brutalement sans pour cela conduire à une trop grande largeur d'ouvrage; leur hauteur est en effet imposée par la marge à prendre vis-à-vis du tirant d'eau à l'aval (photos $\mathrm{n}^{\text {s }} 5,6,10,11$ ).

\section{c) Fonctionnement du dispositif de dispersion.}

Les essais réalisés sur ce dispositif ont montré son aptitude à remplir les conditions imposées pour l'évacuation des crues.

Les jets bien diffusés dans l'air donnent un bon étalement de la zone d'affouillement (la fosse se situe à $60 \mathrm{~m}$ des ouvrages et à une largeur d'environ $30 \mathrm{~m}$ pour un débil de $830 \mathrm{~m}^{3} / \mathrm{s}$ réparti sur les trois pertuis, ou sur le pertuis central seul). Il n'y a pas, jusqu'à un débit de $1.000 \mathrm{~m}^{3} / \mathrm{s}$, de risques d'attaque des berges soit par action directe des jets, soit par courant de retour.

D'autre part, quand la barre formée par les affouillements n'est pas trop importante (barre formée par un débit de 800 à $1.000 \mathrm{~m}^{3} / \mathrm{s}$ ) le fonctionnement d'un quelconque des trois pertuis empêche toute rentrée d'eau et permet de maintenir les autres galeries à sec.

Si, par suite du fonctionnement des galeries de vidange, le plan d'eau s'établit à l'aval audessus du radier de l'évacuateur (tirant d'eau d'environ $1 \mathrm{~m}$ ) la mise en service d'une quelconque des galeries assure rapidement la vidange des deux autres, facilitant ainsi leur fonctionnement éventuel.

Enfin, si une barre importante s'est formée à l'aval de l'évacuateur, il peut $y$ avoir déversement par-dessus les becs et les dents latérales. Lorsqu'un quelconque des pertuis fonctionne, l'aspiration créée par l'écoulement de la galerie permet de vider le débit passant par-dessus le dispositif de dispersion en n'y laissant qu'un faible tirant d'eau.

Le phénomène est mis en évidence sur les photos $\mathrm{n}^{\circ \mathrm{s}} 8$ et 9 et $\mathrm{n}^{\circ \mathrm{s}} 12,13$ et 14 .

Lorsque (photos $n^{\text {os }} 6$ et 10) le tirant d'eau est inférieur à la cote 463,50 (cote minima des arêtes des becs) soit $2,75 \mathrm{~m}$ au-dessus du radier aval de l'évacuateur de crues, aucune rentrée d'eau dans les pertuis non en service n'est à craindre.

Sur la photo $n^{\prime \prime} 7$ où le pértuis central évacue seul $830 \mathrm{~m}^{3} / \mathrm{s}$ le niveau de la surface libre est compris entre les cotes 463,50 et 464,25 ; il y a un début de déversement à l'extrémité du bec. 


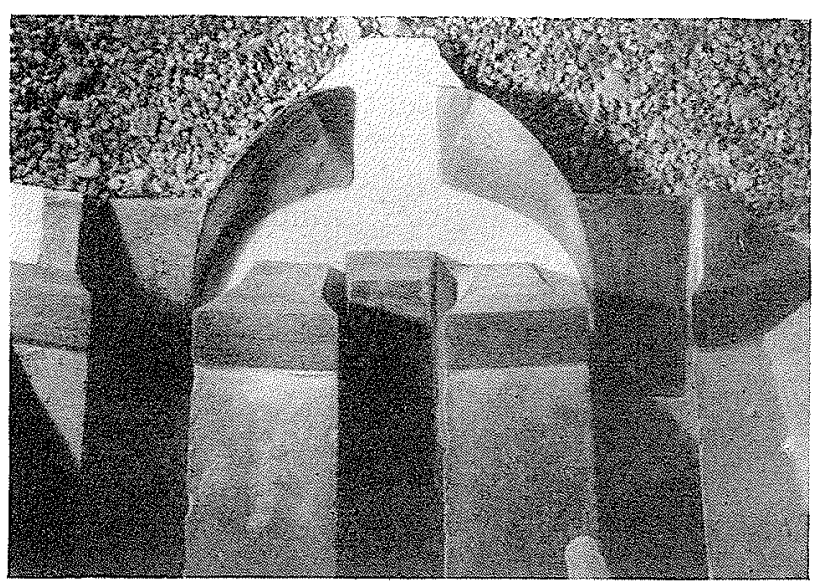

Photo $x^{\circ}$ 3. - Vue de dispositif de pisplersion.

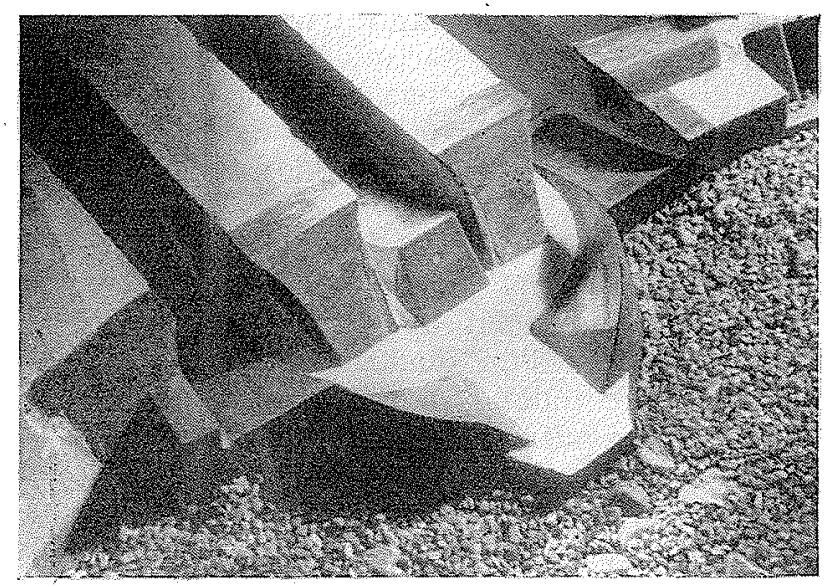

PHOTO $x^{\circ} 4$ - -. VUE DE DispostTtF DE DISPERSON.

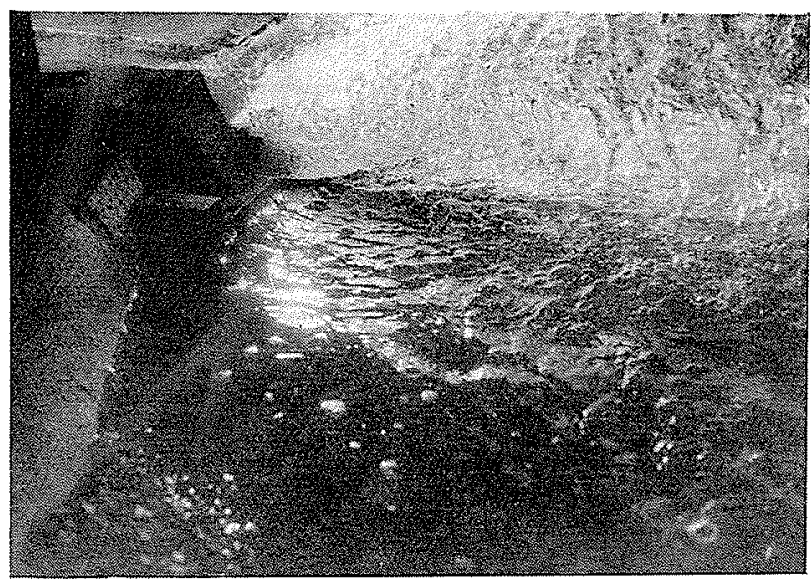

PHOTO $N^{\circ} 5 .-$ DÉBIT DE $830 \mathrm{M}^{3} / \mathrm{SEC}$ PAR LE PERTUIS CENTRAL.

Niveau aval au-dessous de l'arête du dispositif de dispersion.

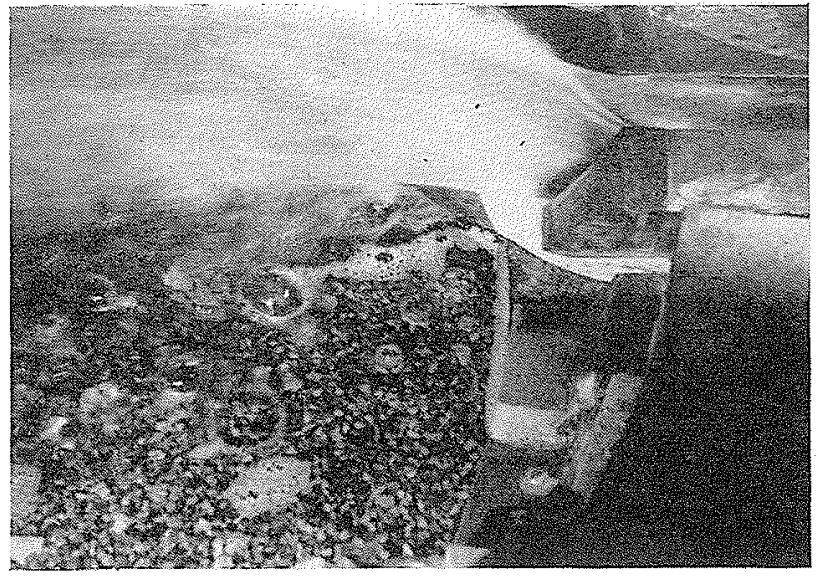

PHOTO N"6.-DIRECTYON DES FILETS LIQUIDES.

Débit de $830 \mathrm{~m} 3 / \mathrm{sec}$ dans le pertuis central.

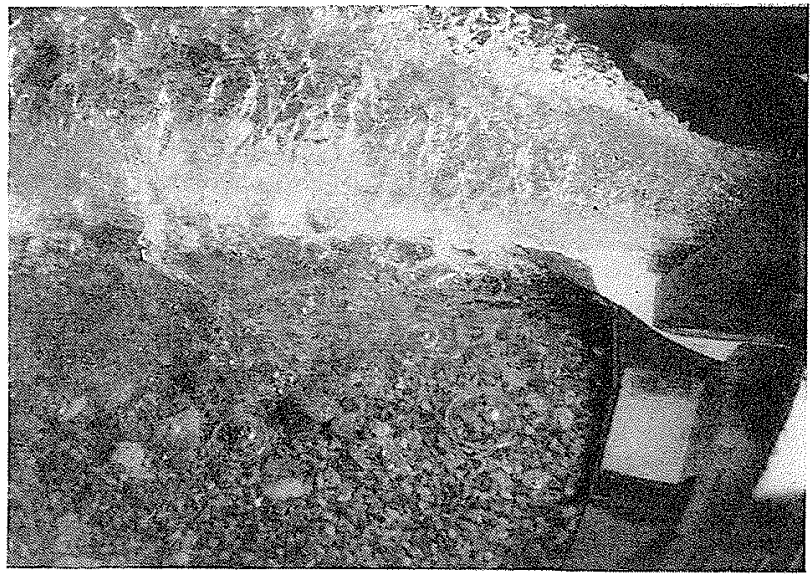

Photo $x^{\circ} 7 .-$ Début DE DEversement A L'EXTREMTTE DE BEC.

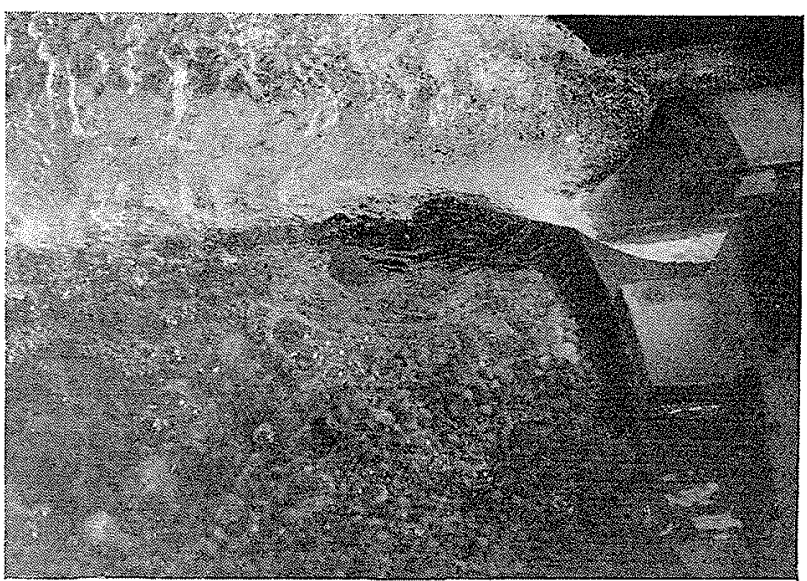

PHOTO $x^{\circ} 8$. DÉversement SUR TOUTE L'ARÉte DU BEC. 


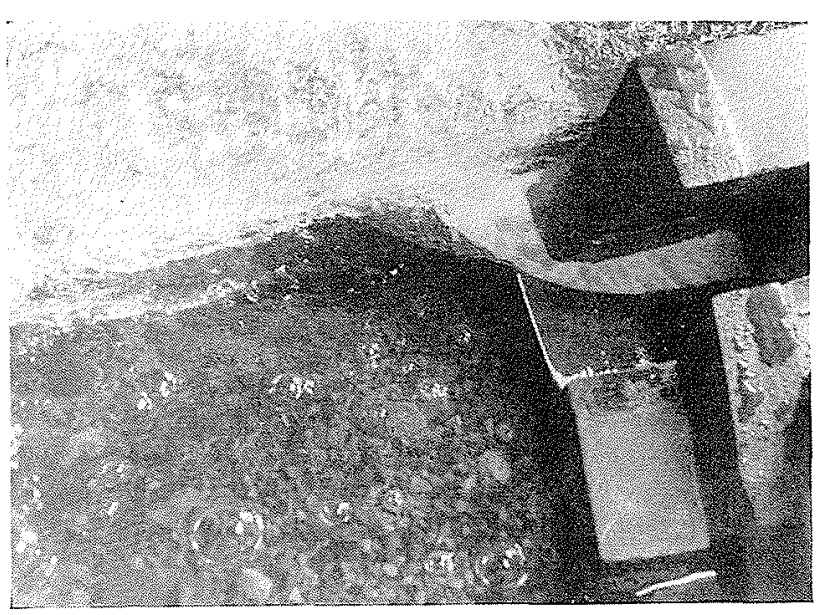

Photo $x^{\prime \prime} 9$. DÉversenen't SUR L'ARÉte DU BEC ET DEE LA DENT.

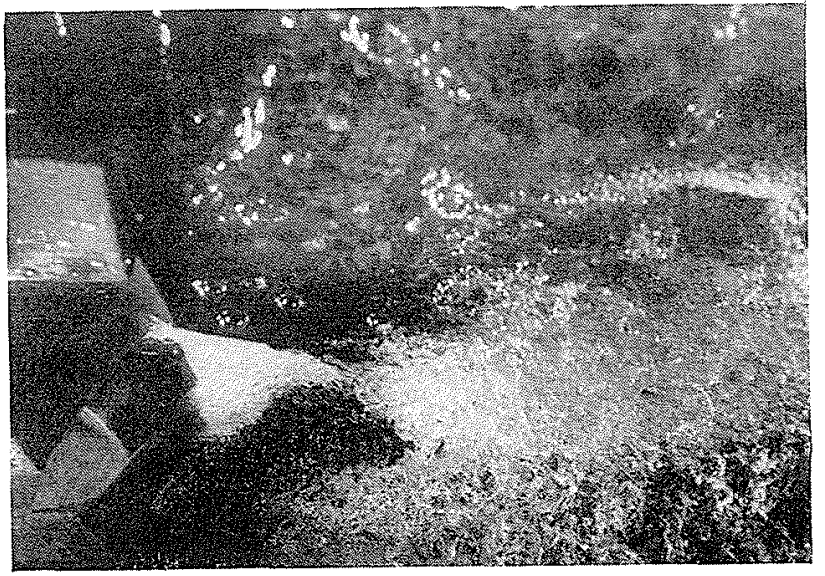

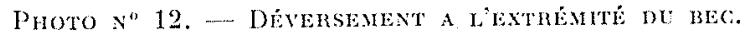

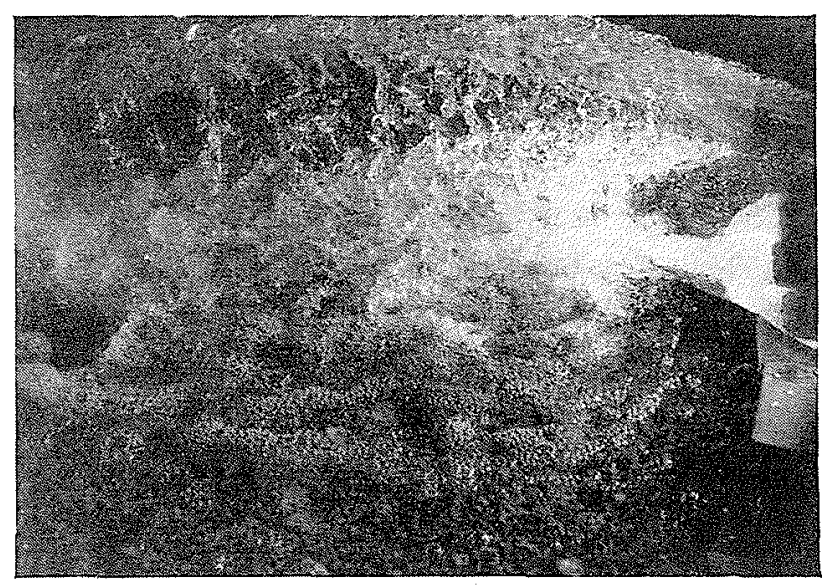

Photo $N^{\circ} 10$, - Vue gínérale dU JET. $830 \mathrm{~m}^{3} / \mathrm{sec}$ dans le pertuis rive droite.

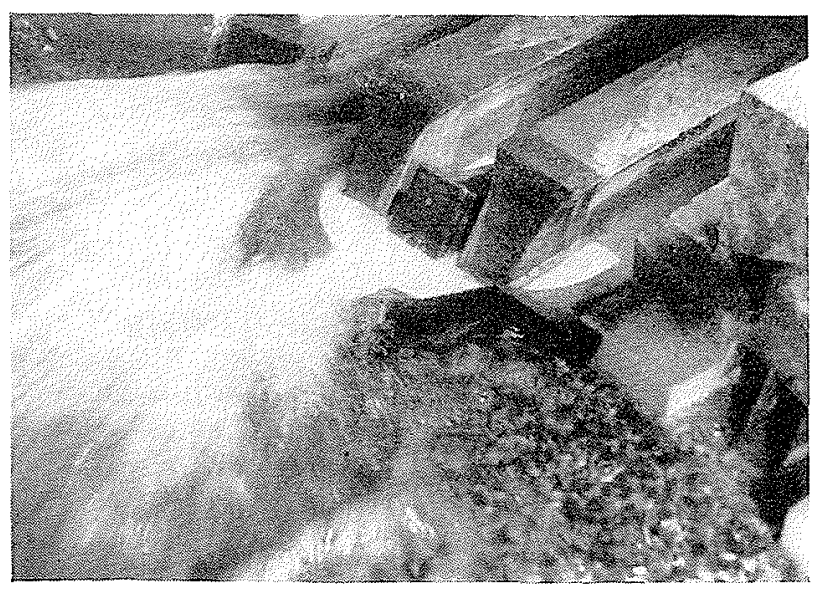

Photo $x " 11$ - ECOULEMENT DE $830 \mathrm{M} 3 / \mathrm{seC}$ DANS LE PERTUIS RIYS DROITE. Direction des filets liquides.

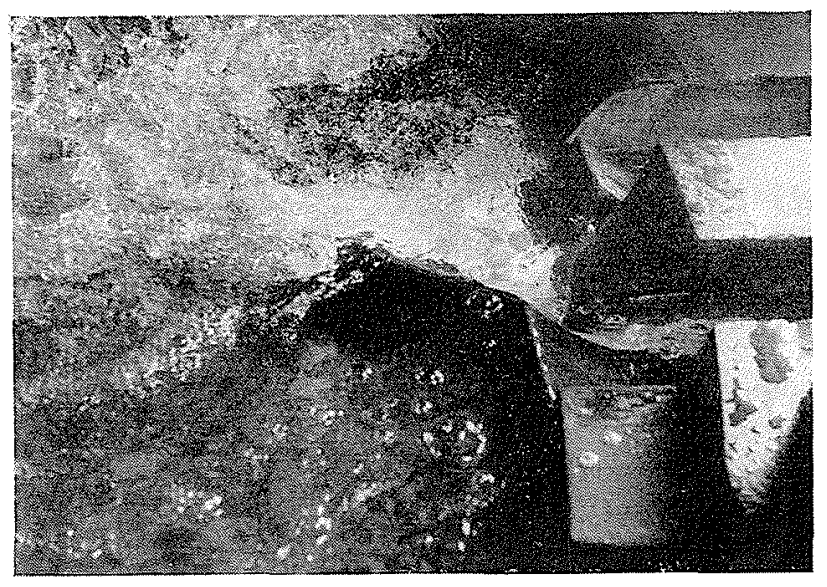

PHOTO N" 13 . - DÉvERSEMENT SUR L'ARETTE DU BEC ET DÉBUT DE DÉYERSEMENT SUR LA DENT.

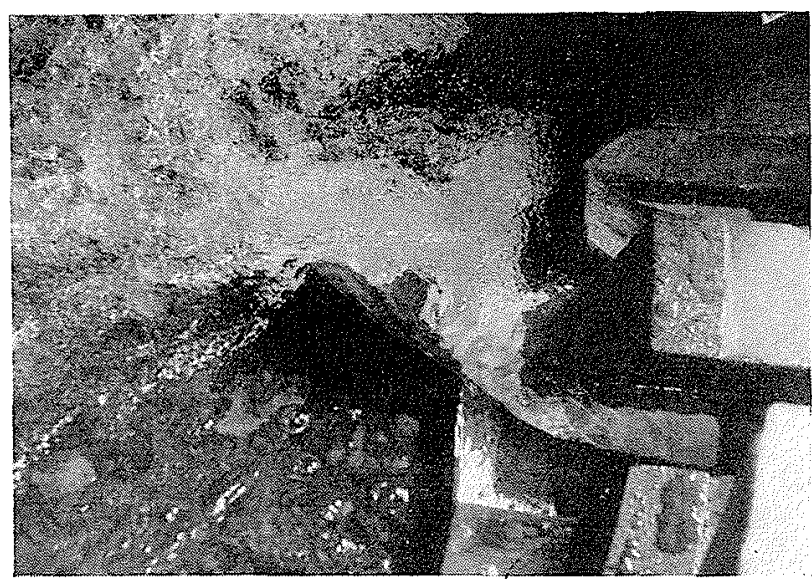

Photo N" 14 . - DÉversemint sur TOUTE r'Arête DU DISPOSITIF DE DISPERSION. 
Il en résulte un très faible tirant d'eau dans les galeries rive droite et rive gauche de l'ordre de $0,20 \mathrm{~m}$ (cote 460,70 ).

Sur la photo $\mathrm{n}^{\circ} 8$, la cote aval du plan d'eau est de 464,25 : il y a déversement sur toute l'arête du bec sans qu'il y ait déversement sur l'arête de la dent dont la cote est précisément 464,25. Le tirant d'eau dans les galeries rive droite et rive gauche est d'environ 0,40 soit à la cote 460,90 .

Enfin, sur la photo $\mathbf{n}^{\circ} 9$, le plan d'eau à l'aval est supérieur à la cote 464,25 , et de l'ordre de 464,75 . Le tirant d'eau dans le dispositif de dispersion est de $1,50 \mathrm{~m}$ environ soit à la cote 463 .

Les photos $\mathrm{n}^{\circ \mathrm{s}} 12,13,14$ mettent en évidence le même phénomène quand le pertuis rive droite est seul en fonctionnement (débit de $830 \mathrm{~m}^{3} / \mathrm{s}$ ).

Il existe donc une certaine relation entre le niveau aval de l'eau dans la vallée, et le tirant d'eau dans les galeries non en service par suite de l'aspiration créée par le jet provenant du pertuis ouvert.

\section{d) Calcul de l'effet d'injection par le jet.}

Cette relation peut être calculée en s'inspirant de la théorie de Tollmien relative à l'épanouissement d'un jet fluide plan dans un milieu fluide indéfini.

Rappelons brièvement les hypothèses à la base de cette théorie.

Considérons un jet plan uniforme de largeur $A B$ (fig. 6) et faisons abstraction des couches limites dues aux parois en amont de AB. Nous pouvons diviser ce jet en deux régions :

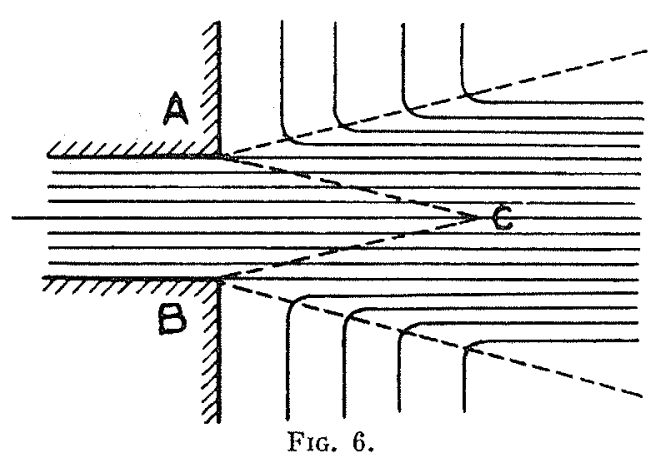

a) Une première où l'on se trouve dans le cas de deux fluides de vitesses différentes (l'une étant nulle) le long de leur frontière commune qui s'épanouit sous un angle constant; la distribution transversale des vitesses moyennes locales, discontinue en $A$, prend un aspect continu à l'aval. b) Une deuxième région située au-delà du point $\mathrm{C}$ où la largeur du jet initial disparaît dans l'épanouissement du jet, et la répartition des vitesses moyennes locales se fondent dans des courbes en cloche.

\section{Méthode de Calcul}

Introduisons la longueur de mélange $l$ à la manière de Prandtr (fig. 7), en utilisant les notations habituelles.

$$
\tau_{l}(x, y)=\rho^{2}\left|\frac{d \bar{u}}{d y}\right| \frac{d \bar{u}}{d y}
$$

$\mathrm{Et}$, comme on constate que la largeur totale de brassage $\mathrm{L}$ est proportionnelle à la distance à l'origine du brassage, posons comme hypothèse :

$$
l=\lambda \mathrm{L} \quad \text { d'où } \quad l=c x,
$$

c résulte de l'expérience.

A la frontière libre, l'élargissement linéaire conduit à poser :

$$
\bar{u}=f\left(\frac{y_{1}}{x}\right)=f\left(r_{1}\right) \quad \text { en posant } \quad \eta=\frac{y}{x}
$$

d'où une fonction de courant :

$$
\psi=\int^{y} f\left(\frac{y}{x}\right) d y=x \mathrm{~F}(n)
$$

la suite des calculs montre que $F$ satisfait à l'équation différentielle :

$$
\mathrm{F}+2 c^{2} \mathrm{~F}^{\prime \prime \prime}=0
$$

en posant :

$$
\begin{gathered}
\eta=\xi \sqrt[3]{2 c^{2}} \\
\mathrm{~F}=c_{1} e^{-\xi}+c_{2} e^{\xi / 2} \cos \frac{\sqrt{3}}{2} \xi+c_{3} e^{\xi / 2} \sin \frac{\sqrt{3}}{2} \xi
\end{gathered}
$$

\section{CondrTIONS AUX LIMITES}

Au-delà de $\mathrm{L}_{1}\left(\eta=\eta_{1}\right)$, le fluide n'est pas perturbé; à la limite $\mathrm{L}_{2}\left(\eta=\eta_{2}\right)$, l'aspiration crée un mouvement secondaire parallèle à OY. Ces con- 
ditions permettent de calculer $c_{1}, c_{2}, c_{3}$ en fonction de $\eta_{1}$ et de calculer ensuite $\eta_{11}$ et $\eta_{2}$, si $c$ est donné.

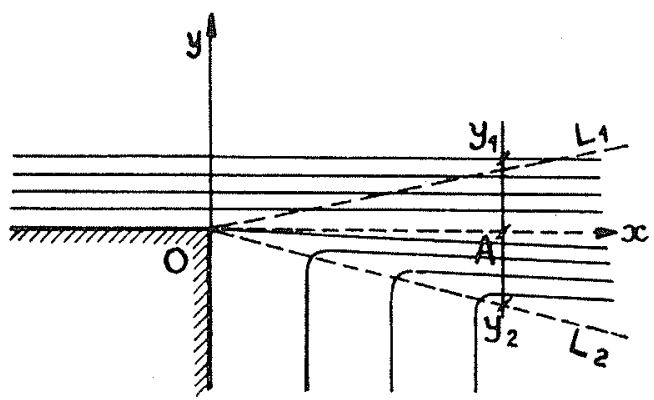

Fig. 7.

Tollmien, en comparant le calcul de la distribution des carrés $\bar{u}^{2}$ en fonction de $\xi$ et la distribution expérimentale des pressions dynamiques a déterminé le rapport :

$$
\sqrt[3]{2 c^{2}}=\frac{1}{\xi}
$$

et il trouve $\mathrm{c}=0,0174$. La répartition des vitesses $\bar{v}$ est donnée par la figure 8 ci-dessous. A la frontière $L_{2}$, on $a$ :

$$
\xi_{2}=-2,04 \quad \text { et } \quad \frac{v}{V \sqrt[3]{2} \bar{c}^{2}}=0,4 \text {. }
$$

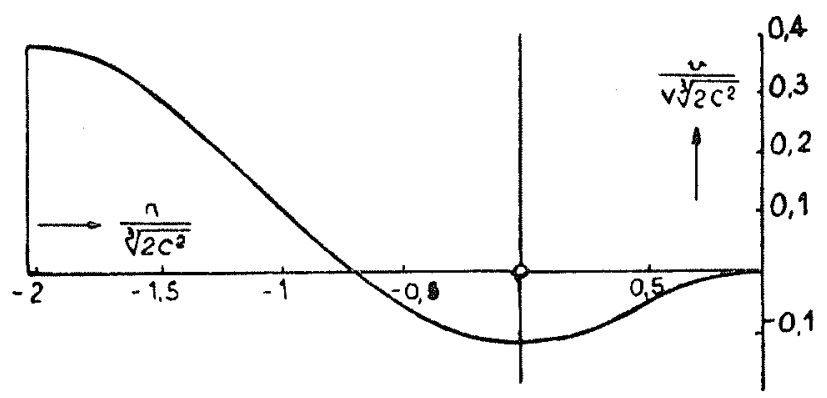

Fic. 8.

On peut remarquer, au sujet de cette théorie, qu'il n'est pas nécessaire de faire intervenir la lóngueur de mélange. En effet, si on reprend le schéma précédent du jet plan s'épanouissant dans un liquide immobile, on voit que le débit $Q$ dans une section $A B$ est égal à $Q=Q_{1}+Q_{2}$, $Q_{1}$ étant le débit passant dans la section $A C$, et $\mathrm{Q}_{2}$ le débit passant dans la section $\mathrm{BC}$ (fig. 9).

Si on appelle $Q_{0}$ le débit passant dans la section (1) et si on admet que le jet s'élargit sous un angle constant (ce qui revient à admettre que $\bar{u}$ est de la forme $\bar{u}=f\left(\frac{y}{x}\right)$, , les largeurs BC et AC étant proportionnelles à $x$, on peut mettre $\mathrm{Q}$ sous la forme $\mathrm{Q}=\mathrm{Q}_{0}+\alpha h \mathrm{~V} x-\beta h \mathrm{~V} x$, $\approx$ et $\beta$ constantes sans dimensions, $V$ étant la vitesse en amont de la section (1). D'où la vitesse $v$ parallèle à $\mathrm{O} y$ à la frontière libre :

$$
\frac{d Q}{d x}=(\alpha-\beta) h V=v h
$$

d'ò̀ :

$$
y=v
$$

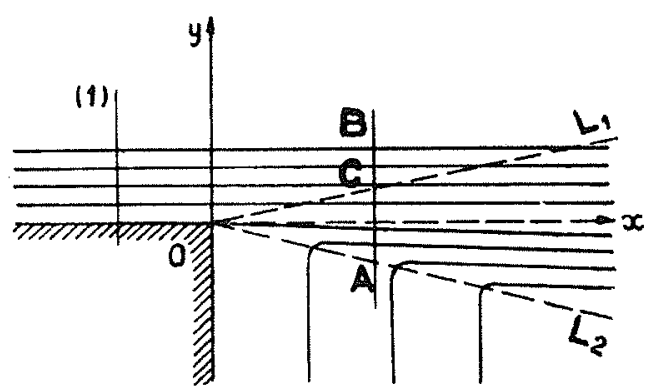

Fic. !.

La valeur de la constante sans dimension $\gamma$ est donnée par l'expérience, comme la constante de Tollmien $\sqrt[3]{2} \overline{c^{2}}$ a été déterminée par ses expériences.

Pour appliquer cette théorie au cas où le pertuis central fonctionnant seul crée une aspiration dans les galeries rive droite et rive gauche, nous supposons, ce qui est une approximation acceptable, que la vitesse $\vec{v}$, parallèle à $O y$, ne dépend pas du tirant d'eau qui baigne le jet. Ce tirant d'eau varie de 0 à $4,00 \mathrm{~m}$, la hauteur du jet étant de l'ordre de $10 \mathrm{~m}$ à la sortie des galeries, dans la zone où se fait sentir l'aspiration. Ceci revient à supposer que l'angle d'épanouissement du jet reste constant; mais il faut remarquer que l'aspiration latérale créée par le jet n'intéresse qu'une longueur $b$ faible du jet $(10 \mathrm{~m})$, où l'on peut considérer $\mathrm{V}$, et par conséquent $v$, comme constants; la valeur de $v$ est donnée par la formule :

$$
\frac{v}{\mathrm{~V} \sqrt[3]{2} c^{2}}=0,4
$$

Dans le cas considéré $\mathrm{V}=28 \mathrm{~m} / \mathrm{s}$, vitesse moyenne correspondant à un débit de $830 \mathrm{~m}^{3} / \mathrm{s}$ s'écoulant dans un pertuis en tenant compte 


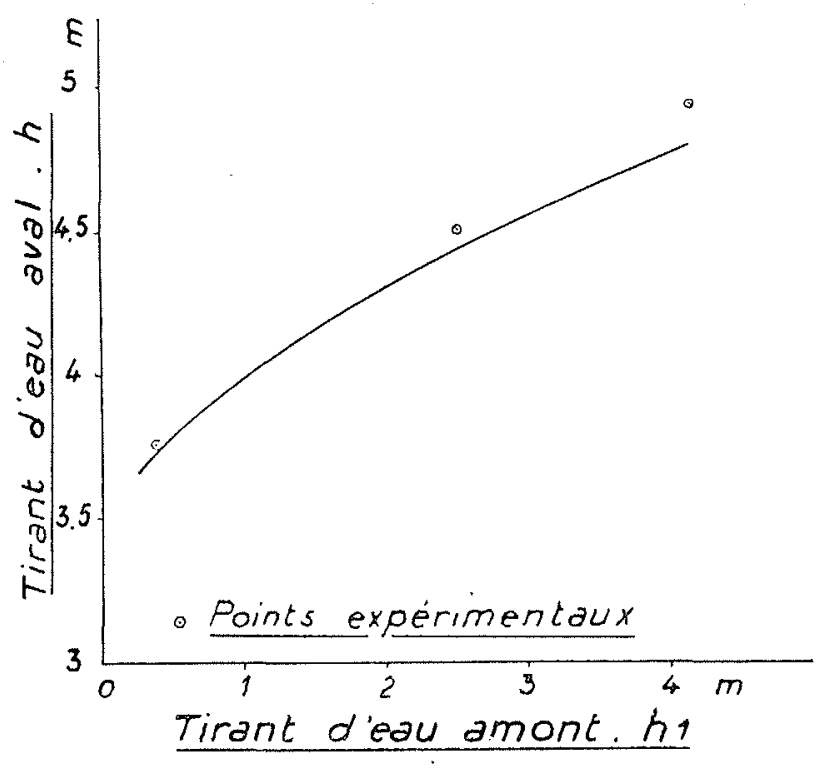

Fifi. 10 . du tiranl d'eau mesuré sur le modele, et $v=0,95 \mathrm{~m} / \mathrm{s}$. A chaque niveau aval $h$ correspond un débit donné q revenant par déversement à l'intérieur du dispositif de dispersion, où le tirant d'eau $h_{1}$ est fonction de $v$ :

$$
l_{1} \#(y / b)
$$

La figure 10 montre la courbe thérique déduite des considerations ci-dessus el les points expérimentaux relevés sur le modele. Ces points correspondent approximativement aux photos $\mathrm{n}^{\mathrm{Ni}} 12,13,14$.

Cette possibilite de maintenir à une cote encore acceptable lo niveau d'cau dans les galeries non en service, fuand il y a déversement a permis de réduire la hauteur des becs, et de réaiser un ouvrage beaucoup moins massif que s'il avait fallu empècher tout risque de deversement a l'intérieur du dispositif de dispersion.

\section{CONCLUSION}

L'aptitude du dispositif à limiter le tirant d'eau dans les galeries non en service, confirmé par la bonne concordance entre la theorie et les mesures sur le modele, montre que, selon toute vraisemblance, lefret d'injection se produira de la mème façon sur louvrage réel, et facilitera ainsi grandement le fonclionnement de l'évacuatenr do erues.
On peul donc espérer que, dans l'ensemble des as concrets, un dispositil de dispersion basé sur un tel principe peut être utilisé toutes les fois où, des galeries débouchant à l'aval à une cote inférieure au plan d'eau dans la vallée, on veut éviter néanmoins la formation d'un ressaut risquant de mettre ces galeries en charge.

\section{I S CUS I O N}

M le President remercie $M$. Chabert et lui demande si les compléments tineoriques permettraient de résoudre de nouveaux problèmes d'évacuateurs de crue avee une confiance absolue dans la théorie, on si l'on deviait encore avoir recours au modèle.

M. Chaberer eroit que l'effet d'aspiration dû au jet provenant d'une galerie s'effectuera bien comme il est prévu dans la théorie, mais que l'étude de la réecption des jets à l'aval peut nécessiter des essais sur modèle récluit, en raison de conditions locales particulières, protection de l'unc ou lautre berge par exemple.

M. le président conclut que la difference entre la théorie el l'expérience réside dans le fait que le problème est dans un cas a deux dimensions, et dans l'autre a trois dimensions. 